\title{
Correction: A novel derivative of betulinic acid, SYK023, suppresses lung cancer growth and malignancy
}

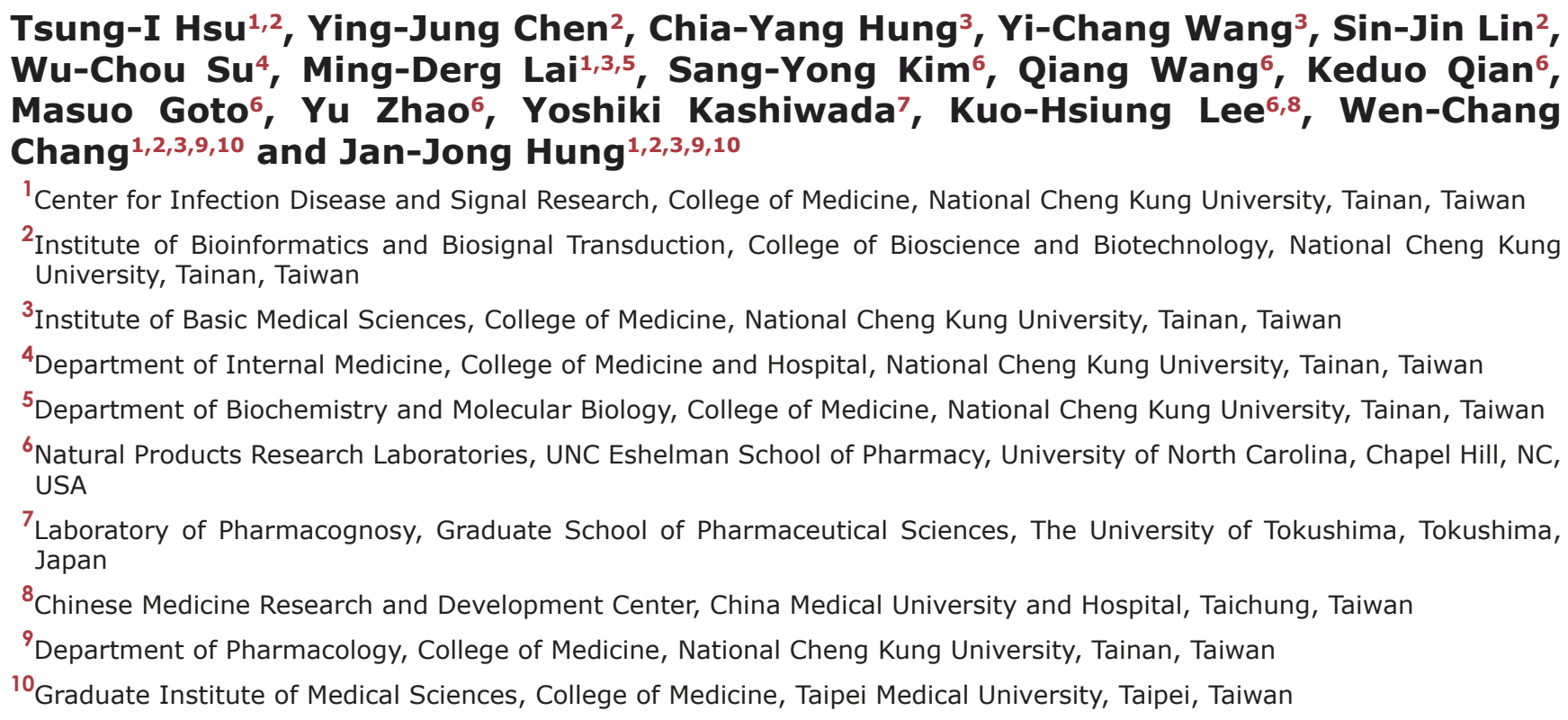

${ }^{1}$ Center for Infection Disease and Signal Research, College of Medicine, National Cheng Kung University, Tainan, Taiwan

${ }^{2}$ Institute of Bioinformatics and Biosignal Transduction, College of Bioscience and Biotechnology, National Cheng Kung University, Tainan, Taiwan

${ }^{3}$ Institute of Basic Medical Sciences, College of Medicine, National Cheng Kung University, Tainan, Taiwan

${ }^{4}$ Department of Internal Medicine, College of Medicine and Hospital, National Cheng Kung University, Tainan, Taiwan

${ }^{5}$ Department of Biochemistry and Molecular Biology, College of Medicine, National Cheng Kung University, Tainan, Taiwan

${ }^{6}$ Natural Products Research Laboratories, UNC Eshelman School of Pharmacy, University of North Carolina, Chapel Hill, NC, USA

${ }^{7}$ Laboratory of Pharmacognosy, Graduate School of Pharmaceutical Sciences, The University of Tokushima, Tokushima, Japan

${ }^{8}$ Chinese Medicine Research and Development Center, China Medical University and Hospital, Taichung, Taiwan

${ }^{9}$ Department of Pharmacology, College of Medicine, National Cheng Kung University, Tainan, Taiwan

${ }^{10}$ Graduate Institute of Medical Sciences, College of Medicine, Taipei Medical University, Taipei, Taiwan

Published:

Copyright: Hsu et al. This is an open-access article distributed under the terms of the Creative Commons Attribution License 3.0 (CC BY 3.0), which permits unrestricted use, distribution, and reproduction in any medium, provided the original author and source are credited.

This article has been corrected: The control data used in the assembly of Figure 5 was incorrect. After reviewing all of the raw data, an updated Figure 5 was created and is shown below. The authors declare that these corrections do not change the results or conclusions of this paper.

Original article: Oncotarget. 2015; 6:13671-13687. https://doi.org/10.18632/oncotarget.3701 


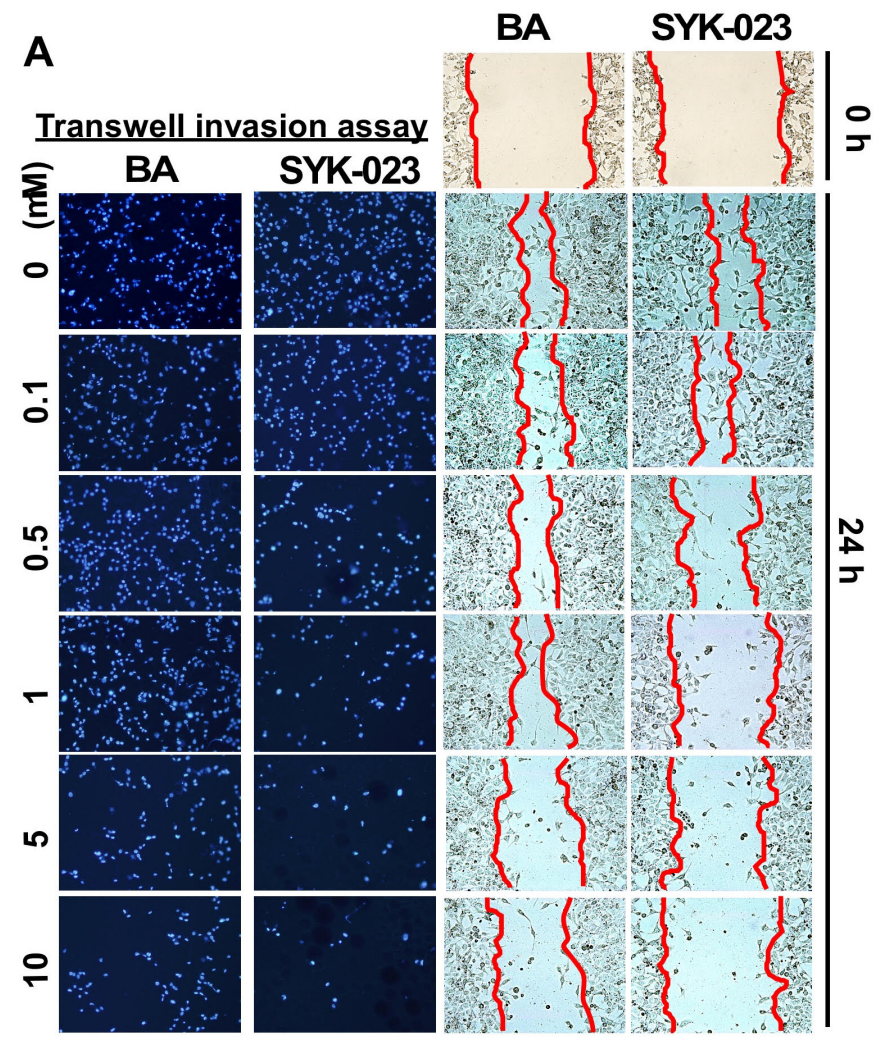

B(a)

更

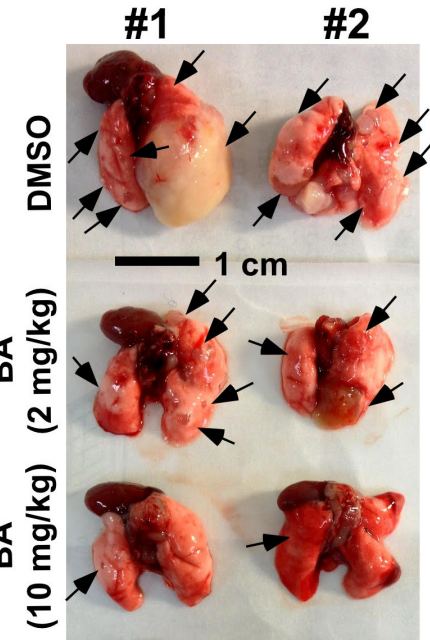

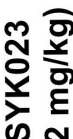

จิ

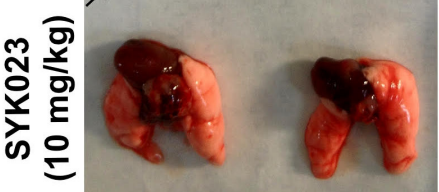

C F-actin/DAPI
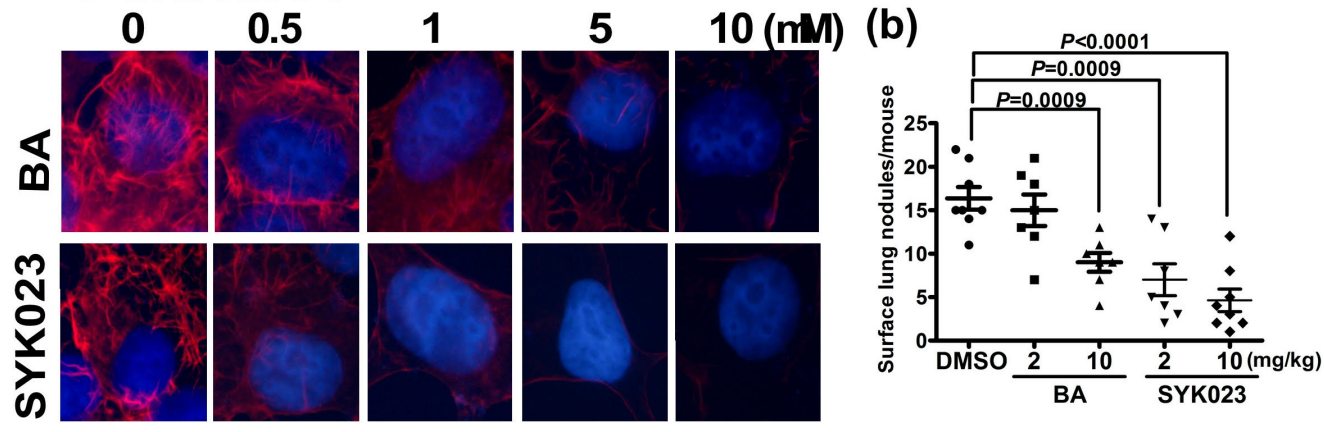

Figure 5: Effects of BA and SYK023 on lung tumor metastasis in vitro and in vivo. (A) After BA and SYK023 treatment for 36 h, H1299 cells were subjected to transwell invasion and wound-healing assay. (B) Representative images of lungs from SCID mice with metastasis (a). The number of surface lung tumors. Data are expressed as mean \pm s.e.m, $P$-value is indicated (b). (C) H1299 cells on the coverslip were treated with the indicated drug, and subjected to immunofluorescent staining for F-actin and DAPI (1000×). 